\title{
Detección de Clorpirifos en Leche usando un Biosensor Enzimático Amperométrico basado en Acetilcolinesterasa
}

\author{
Jacqueline Betancur (1), Duvan F. Morales ${ }^{(2)}$, Gustavo A. Peñuela(1), Juan B. Cano ${ }^{(2)}$ \\ (1) Grupo diagnóstico y control de la contaminación (GDCON), Facultad de Ingeniería, Sede de Investigación \\ Universitaria (SIU), Universidad de Antioquia, Calle 70 No 52-21, Medellín, Colombia \\ (2) Grupo manejo eficiente de la energía (GIMEL), Facultad de Ingeniería, Sede de investigación universitaria \\ (SIU), Universidad de Antioquia, Calle 70 No 52-21, Medellín, Colombia. \\ (e-mail:jabebu15@gmail.com; bernardo.cano@udea.edu.co)
}

Recibido Mar. 14, 2018; Aceptado May. 18, 2018; Versión final Jun. 20, 2018, Publicado Dic. 2018

\begin{abstract}
Resumen
Este trabajo explora el uso de un biosensor enzimático amperométrico, como una herramienta de análisis capaz de determinar la concentración de clorpirifos en leche cruda de vaca, comparado en paralelo con los resultados obtenidos con la técnica de cromatografía de gases. Se utilizó como elemento biológico la enzima acetilcolinesterasa, la cual fue inmovilizada sobre electrodos de diferente material de tipo pantalla impresa (screen printed) mediante el método de union cruzada (cross linking). Se determinó las constantes cinéticas de la enzima tanto libre como inmovilizada. Se realizó la validación de la técnica para la cuantificación de clorpirifos mediante cromatografía de gases, varios parámetros de optimización fueron valorados para el uso del biosensor y se realizó la validación de este como herramienta para detectar clorpirifos en leche. Este trabajo concluye que el biosensor amperométrico es una herramienta útil para la determinación cualitativa de clorpirifos en leche en tiempo real.
\end{abstract}

Palabras clave: colinesterasa; biosensor; clorpirifos; amperometría; leche, prototipo electrónico.

\section{Detection of Chlorpyrifos in Milk using an Amperometric Biosensor based on Acetylcholinesterase}

\begin{abstract}
This work explores the use of an enzymatic amperometric biosensor, as an analysis method capable of determining the concentration of chlorpyrifos in raw cow's milk, compared in simultaneous runs with the results obtained with the gas chromatography technique. The enzyme acetylcholinesterase was used as a biological element, which was immobilized on electrodes of different material of the screen-printed type by the crosslinking method. The kinetic constants of the enzyme, free and immobilized, were determined. Validation of the technique for the quantification of chlorpyrifos by gas chromatography was performed, several optimization parameters were evaluated for the use of the biosensor and the validation of this as a tool to detect chlorpyrifos in milk was done. This paper concludes that the amperometric biosensor is a useful tool for the qualitative determination of chlorpyrifos in milk in real time.
\end{abstract}

Keywords: cholinesterase; biosensor; chlorpyrifos; amperometry; milk, electronic prototype. 


\section{INTRODUCCIÓN}

Los plaguicidas han sido usados para el control de plagas en la agricultura y a plagas que afectan de manera directa al ser humano (Whitford et al., 2009). En Colombia, el Clorpirifos es un insecticida muy usado en la protección de cultivos, pastos para ganado y sitios urbanos. El clorpirifos actúa como un inhibidor de la enzima acetilcolinesterasa lo que produce acumulación del neurotransmisor acetilcolina en las sinapsis mediadas por este, tanto en los insectos como en los mamíferos, afectando directamente el sistema nervioso central (Buck, et al., 1981), (Rodríguez et al., 2013). La exposición de los animales al clorpirifos se presenta mayormente al fumigar los pastos, quedando residuos del plaguicida en las plantas y en el suelo, de donde puede ingresar a los animales por la boca, los pulmones y la piel (Ferri et al., 2014). Después de ser ingerido, pasa rápidamente de los intestinos al torrente sanguíneo, el cual lo distribuye al resto del cuerpo (Buck, et al., 1981), (Rodríguez et al., 2013). En rebaños lecheros el mal uso del clorpirifos puede conducir a la producción de leche con residuos del plaguicida (Morales et al., 2010).

Colombia es un país altamente lechero, cuya producción en el año 2015 fue de aproximadamente tres mil doscientos ochenta y seis millones (3,286 millones) de litros al año (Asoleche, 2017). El clorpirifos, además de ser empleado en los cultivos también es usado para fumigar los pastos para la ganadería. Por esta razón, el clorpirifos debe ser monitoreado en la leche para garantizar al consumidor un producto inocuo para la salud de las personas. Según la organización mundial de la salud (OMS), la concentración límite permitida de clorpirifos en leche de vaca para no afectar la salud humana es de 0.02mg/L (Food Standars FAO/WHO, 2013). Las técnicas más utilizadas para la detección y cuantificación del clorpirifos en alimentos son la cromatografía líquida de alta eficiencia y la cromatografía de gases. Estas técnicas de análisis requieren de procedimientos con largos tiempos de preparación de las muestras. Estas técnicas son inviables para ser usadas en el campo donde se tiene el ganado.

Recientemente, los biosensores han emergido como complemento a las técnicas tradicionales de análisis. Estos emplean un material biológico (una enzima, un tejido, un anticuerpo, etc.) como elemento principal de sensado. En la literatura, se han reportado aplicaciones en los campos: médico (Hernández et al., 2011) (Montañez et al., 2011), espacial (Cano et al., 2011), seguridad alimentaria (Lavecchia et al., 2010) y protección ambiental (Scognamiglio et al., 2009). Los biosensores se han empleado como como herramientas de análisis capaces de detectar la presencia de clorpirifos en leches en tiempo real (Scognamiglio et al., 2012) (Silleti et al., 2015) lo que es una gran ventaja para la industria lechera, ya que permite rechazar leches en el mismo hato que tengan presencia de clorpirifos.

En este trabajo se describe el desarrollo de un biosensor basado en la enzima acetilcolinesterasa para la detección de clorpirifos directamente en hatos lecheros. Este tipo de sensor ha sido reportado en la literatura científica pero poco explorado en aplicaciones prácticas en Colombia y Latinoamérica. Mediante el uso del biosensor se logra detectar concentraciones de clorpirifos por debajo del valor exigido por la OMS (20 $\mu \mathrm{g} / \mathrm{L})$ como una alarma al incumplimiento de la normatividad exigida en la industria de alimentos y específicamente en la industria láctea. Trabajos similares logran detectar concentraciones de pesticidas organofosforados por encima de $1000 \mu \mathrm{g} / \mathrm{L}$ (Rodríguez et al., 2013; Morales et al., 2010) lo que no aporta mucha importancia a la hora de cumplir con los parámetros de calidad. Se realiza una descripción y comparación de diferentes tratamientos para la inmovilización del biomediador, la cual no se encuentra reportada en trabajos similares (Scognamiglio et al., 2012), (Arduini et al., 2013) (Rodríguez et al., 2013) y es necesaria para el exitoso desarrollo del biosensor.

Se realizó una verificación del funcionamiento del biosensor según la norma SANTE 11945/2015 (poco reportada en artículos sobre biosensores) y una comparación con la técnica analítica de la cromatografía gaseosa. Esta comparación permite concluir la utilidad del biosensor para la detección cualitativa (más no cuantitativa) para la detección del plaguicida. Adicionalmente se presenta un dispositivo electrónico móvil, pensado para el funcionamiento en campo, con capacidades de interacción con dispositivos celulares vía bluetooth, el cual permitió el desarrollo y validación del biosensor así como su futura aplicación en entornos reales.

\section{MATERIALES Y MÉTODOS}

Inicialmente se describe el prototipo electrónico desarrollado para la medida amperométrica. Luego se describen los procesos relacionados con el biomediador, como son la caracterización de la enzima libre, la inmovilización, la verificación y optimización de su funcionamiento. Finalmente, se describe la técnica de cromatografía de gases que será comparada con el biosensor.

Se desarrolló un prototipo (Figura 1) para realizar medidas de amperometria y voltametría cíclica sobre un sensor impreso (SPE - Screen Printed Electrode) de tres electrodos. El prototipo es de bajo costo, portable, 
fácil de operar y está pensado para permitir la medida en campo de la contaminación en hatos lecheros. El prototipo cuantifica la señal bioquímica producida en la reacción enzimática dada sobre el electrodo previamente inmovilizado y utilizado en las pruebas de inhibición y de optimización. Para el desarrollo se tuvieron en cuenta las consideraciones de diseño electrónico necesarias para garantizar la integridad de las pequeñas señales involucradas en la medida amperométrica (Cano et al., 2014).

Las principales características del dispositivo son: 1) Conexión directa de sensores impresos tipo SPE de la serie DROPSENS; 2) Dos tipos de medida: Voltametría cíclica y cronoamperometría; 3) Potencial de óxidoreducción configurable entre $-1000 \mathrm{mV}$ a $+1000 \mathrm{mV}$, con resolución de $1 \mathrm{mV}$; 4) Rango de medida de corriente configurable entre +/-7uA, +/-70uA, +/-700nA; 5) Variación de voltaje cada 0.5 segundos para la voltametría cíclica; 6) Alimentación desde baterías o desde puerto USB del computador; 7) Posibilidad de comunicación de datos vía USB para uso en laboratorio o vía Bluetooth para uso en campo por medio de dispositivos smartphone - tablet. 8) Aplicación para computador o APP para celular que permiten el control del dispositivo y la recolección de datos.
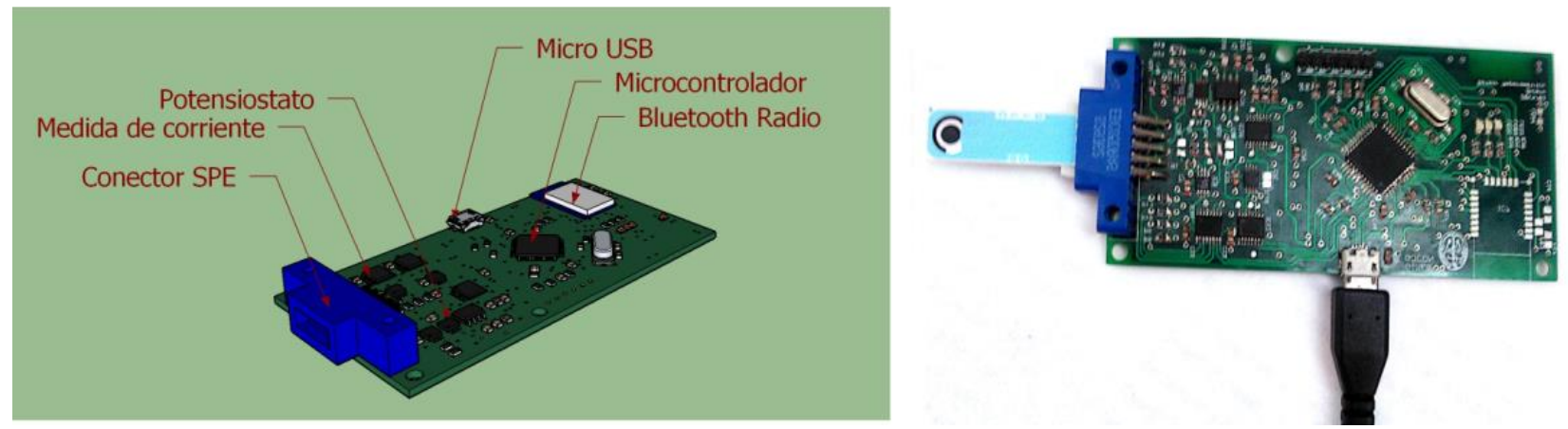

Fig. 1: Izquierda, modelo 3D del prototipo desarrollado con sus principales componentes. Derecha, fotografía del prototipo durante las pruebas del biosensor.

La actividad enzimática de la enzima libre se determinó según el método usado por Ellman et al (1960). Las soluciones preparadas fueron: Buffer fosfato 0.1M; pH 8; Acetiltiocolina (Acetylthiocholine chloride SIGMA, referencia: A5626) para realizar una curva de concentraciones de $0.1 \mathrm{mM}, 0.15 \mathrm{mM}, 0.25 \mathrm{mM}, 0.3 \mathrm{mM}, 0.4 \mathrm{mM}$, 0.5mM, 0.7mM, 0.85mM, $1.0 \mathrm{mM}$; Acetilcolinesterasa $0.01 \mathrm{mg} / \mathrm{mL}$ (SIGMA, referencia: C3389); DTNB 0.01M (5,5'-Dithio-bis-(2-nitrobenzoic Acid) SIGMA, referencia: 322123). Las soluciones fueron preparadas con agua desionizada. La actividad enzimática se hizo con un espectrofotómetro a una longitud de onda de $416 \mathrm{~nm}$. La preparación final para la lectura fue de la siguiente manera: $500 \mu \mathrm{L}$ DTNB $+500 \mu \mathrm{L}$ Sustrato $+950 \mu \mathrm{L}$ Buffer fosfato + $50 \mu \mathrm{L}$ Enzima o $1000 \mu \mathrm{L}$ Buffer fosfato, en el caso del blanco no lleva enzima. Para la evaluación de la inhibición enzimática se usó $0,02 \mathrm{mg} / \mathrm{L}$ de clorpirifos a diferentes concentraciones de sustrato (las mismas utilizadas para la determinación de la actividad cinética sin inhibición), cuyo procedimiento para la lectura mediante espectrofotometría fue 500 $\mu \mathrm{L}$ DTNB $+500 \mu \mathrm{L}$ Sustrato $+900 \mu \mathrm{L}$ Buffer fosfato $+50 \mu \mathrm{L}$ Enzima +50 $\mu \mathrm{L}$ clorpirifos o $950 \mu \mathrm{L}$ Buffer fosfato, en el caso del blanco no lleva enzima. La lectura se hizo por triplicado y se realizó un blanco por cada ciclo de actividad enzimática.

Para la determinación de la velocidad de reacción se empleó la ecuación 1.

$$
V e l \operatorname{rxn}\left(\frac{m m o l}{L} * \min \right)=\frac{\frac{\Delta A b s}{\min } * V e * F}{\varepsilon * l * e n z}
$$

En esta ecuación, Ve es el volumen total del ensayo $(\mathrm{mL}), F$ es el factor de dilución de la enzima, $\varepsilon$ es el coeficiente de extinción molar del cromóforo y $\lceil$ es la longitud de la celda en cm por donde pasa el haz de luz. La Velocidad máxima y la constante de Michaelis-Menten (km) fueron determinadas mediante la representación de Linewaver-Burk, según la ecuación 2.

$$
\frac{1}{V}=\frac{k m}{V m a ́ x[S]}+\frac{1}{V m a ́ x}
$$

La Inmovilización del elemento biológico (acetilcolinesterasa) se llevó a cabo en la superficie del electrodo de trabajo mediante la técnica de cross-linking. Se emplearon Albumina de suero fetal Bovino (BSA) $5 \%-6 \%$ (Albumin bovine serum SIGMA, referencia: A4503); Glutaraldehido (GA) 1-2.5\% (Glutaraldehyde solution SIGMA, referencia: G5882); Acetilcolinesterasa 10U-100U. La inmovilización se evaluó en diferentes tipos de electrodos con cuatro tratamientos: Tratamiento 1: GA 2.5\%; BSA 6\%; 100U AChE; Tratamiento 2: GA 2.5\%; 
BSA 6\%; $10 \mathrm{U}$ AChE; Tratamiento 3: GA 1\%; BSA 5\%; $100 \mathrm{U}$ AChE; y Tratamiento 4: GA 1\%; BSA 5\%; $10 \mathrm{U}$ AChE

Para los tratamientos 1 y 2, la mezcla se preparó con la siguiente relación: $5 \mu \mathrm{L} B S A+5 \mu \mathrm{L} G A+10 \mu \mathrm{L} A C h E$. Se tomaron $5 \mu \mathrm{L}$ de la mezcla y se adicionaron sobre el electrodo de trabajo. Para los tratamientos 3 y 4 , la mezcla se preparó con igual cantidad de BSA, GA y AChE. Se tomó $2 \mu \mathrm{L}$ de la mezcla resultante y fueron adicionados sobre el electrodo de trabajo. Los electrodos se dejaron enfriar a $4^{\circ} \mathrm{C}$ durante $24 \mathrm{~h}$ y almacenados en solución Buffer $1 \mathrm{M}, \mathrm{pH} 8$ a una temperatura de $4^{\circ} \mathrm{C}$ por un periodo de tiempo hasta de 15 días. Cada vez que se realizó la lectura, la enzima se activó a una temperatura de $28^{\circ} \mathrm{C}$. Los cuatro tratamientos fueron evaluados con electrodos de carbono (Dropsens, referencia: 110), oro (Dropsens, referencia: 220BT), platino (Dropsens, referencia: 550), y los tratamientos uno y dos fueron evaluados en electrodos de carbón con potencializadores: carbón con azul de Prusia (Dropsens, referencia: 710) y carbón con ferrocianuro (Dropsens, referencia: F10). Para cada tipo de electrodo se realizó por triplicado Voltametría cíclica con un barrido de voltaje de $-1000 \mathrm{mV}$ a $1000 \mathrm{mV}$, durante 5 ciclos y con una variación de lectura de $20 \mathrm{mV}$ cada 0.5 segundos. Todos los ensayos amperométricos se realizaron con una concentración de sustrato de $5 \mathrm{mM}$.

Para hallar la mejor respuesta del biosensor según la concentración de sustrato, se realizó un barrido de voltaje desde $-1000 \mathrm{mV}$ a $1000 \mathrm{mV}$ con 5 ciclos y una variación de $20 \mathrm{mV}$ cada $0.5 \mathrm{~s}$, por triplicado con diferentes concentraciones de sustrato $(1 \mathrm{mM}, 3 \mathrm{mM}, 5 \mathrm{mM}, 8 \mathrm{mM}, 10 \mathrm{mM})$ en los electrodos de carbono, carbono-azul Prusia y carbono-ferrocianuro. Los ensayos de inhibición del biosensor se realizaron en agua, leche cruda y en leche descremada para evaluar el efecto de la grasa en la medida mediante cronoamperometría. El biosensor como una herramienta de detección del clorpirifos en leche fue validado según los criterios reportados en la guía SANTE 11945/2015.

El porcentaje de inhibición fue determinado según la ecuación 3.

$$
\% I=\frac{I a-I b}{I a} \times 100
$$

En la ecn. (3), Ia es el valor de la corriente registrada de la actividad enzimática para el sustrato, $I b$ es el valor de la corriente registrada de la actividad enzimática una vez adicionado el inhibidor.

La verificación de los resultados con el biosensor se hizo mediante comparación por cromatografía de gases acoplada a un detector de espectrometría de masa (CG/EM). La técnica fue validada según los criterios reportados en la guía SANTE 11945/2015. El pretratamiento de la muestra (clorpirifos) se realizó con el método QuEChERS, reportado en la AOAC 2007.01, usando: acetonitrilo al $1 \%$ con ácido acético, sales de extracción (6 g MgSO4 + $1.5 \mathrm{~g} \mathrm{NaC}_{2} \mathrm{H}_{3} \mathrm{O}_{2}$ ), cartucho de limpieza (SPE) (50 mg PSA + 50 mg C18 + $150 \mathrm{mg}$ MgSO4). Se tomó una alícuota de muestra pretratada para el análisis por CG/EM, usándose una columna HP-5MS UI (Medianamente polar), longitud de $30 \mathrm{~m}$, diámetro de $0.250 \mathrm{~mm}$ y capa de $0.25 \mu \mathrm{m}$. El gas de arrastre fue helio.

\section{RESULTADOS Y DISCUSIÓN}

La determinación de la actividad cinética se realizó mediante espectrofotometría tanto para la enzima libre sin inhibidor como para la enzima libre con inhibidor; para conocer su influencia en el comportamiento de la actividad cinética.

En la figura 2 se muestra el resultado para el seguimiento de la formación de Tiocolina, procedente de la reacción de la Acetiltiocolina en presencia de acetilcolinesterasa (sin inhibidor), durante un intervalo de tiempo de 16 minutos. La hidrólisis de la acetiltiocolina por la acción de la acetilcolinesterasa genera como productos de reacción la Tiocolina y el acetato. La tiocolina liberada reacciona con el cromóforo DTNB, dando lugar a la formación del ácido 5-tio-2-nitrobenzoico (TNB2), compuesto de color amarillo, siendo el valor máximo de absorbancia a una longitud de onda de 412nm (Rodríguez et al., 2013)(Tecles et al. 2000). La tasa de aparición de este producto de reacción es mayor conforme aumenta la actividad enzimática existente en la muestra. A medida que aumenta la concentración de sustrato también incrementa la actividad enzimática. A la concentración de sustrato correspondiente a $0.4 \mathrm{mM}$ todos los valores de la absorbancia durante los 16 minutos evaluados se encuentran por debajo de la unidad, cumpliendo la ley de Lamber-Beer. Según los resultados (figura 2), la medida de actividad enzimática con una concentración de $0.5 \mathrm{mM}$ de acetiltiocolina después de los 12 minutos no es confiable, pues sobrepasa el valor de la unidad para la absorbancia. Se presentó rápidamente una saturación de la enzima, a las concentraciones de acetiltiocolina de $0.7 \mathrm{mM}$, $0.85 \mathrm{mM}$ y $1.0 \mathrm{mM}$. Para hallar las constantes cinéticas tales como Vmáx y km, se tomó los valores de la absorbancia entre 0 y 10 minutos en el rango de concentración de sustrato de 0.1 a $0.5 \mathrm{mM}$. A partir de estos datos se realizó la representación de Linewaver-Burk. Se obtuvo una ecuación de la recta correspondiente a 
$y=0.0203 x+0.0008$, interpretando esto mediante la ecuación 2 , el valor de Vmáx para la enzima acetilcolinesterasa fue $1250 \mu \mathrm{mol} / \mathrm{min}$ y el $\mathrm{km}$ fue $2.54 \mathrm{E}-05 \mathrm{M} / \mathrm{min}$
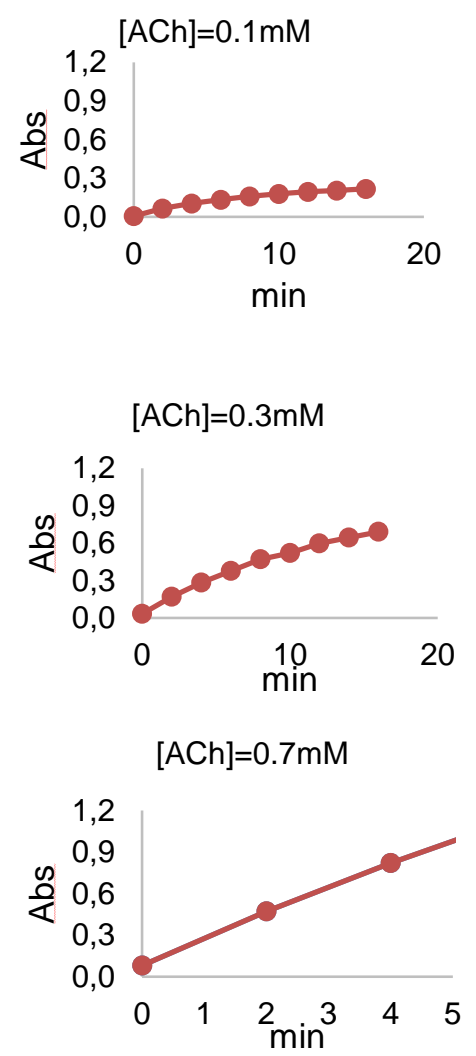
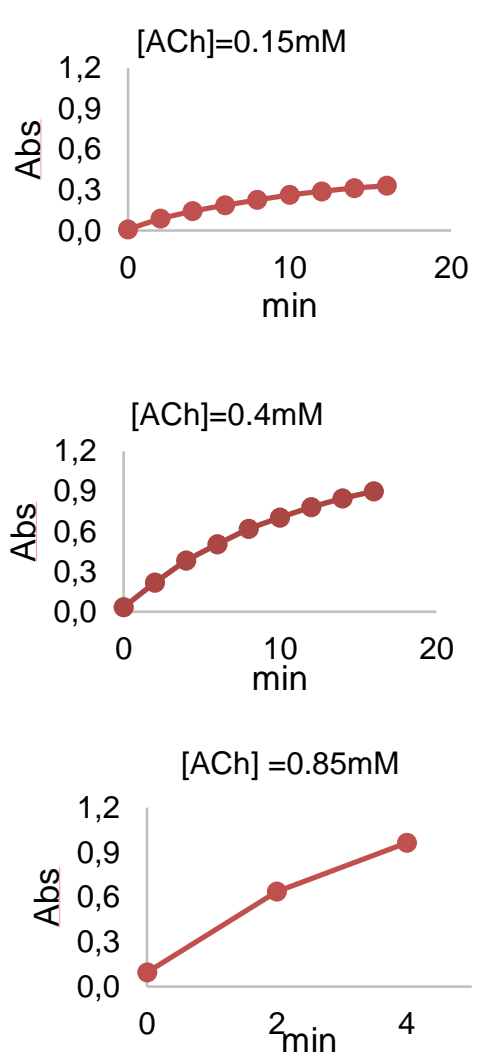
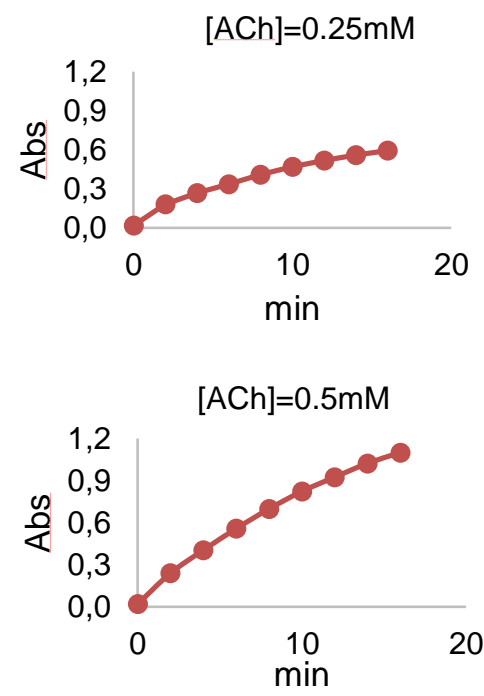

$[\mathrm{ACh}]=1.0 \mathrm{mM}$

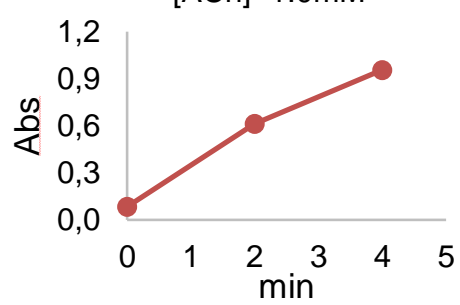

Fig. 2: Actividad enzimática de la acetilcolinesterasa a diferentes concentraciones de sustrato.

Los resultados para la determinación de la actividad enzimática de la enzima libre con inhibidor arrojaron una ecuación de la recta según la representación de Linewaver- Burk y $=0.0361 x+0.1721$. Mediante la ecuación 2 se deduce que los valores de Vmáx(i) y km(i) son respectivamente $5.810 \mu \mathrm{mol} / \mathrm{min}$ y $2.098 \mathrm{E}-07 \mathrm{M} / \mathrm{min}$. Para la enzima libre con inhibidor, $\mathrm{km}$ (i) es más pequeño, lo que significa que la afinidad que presenta la enzima por el inhibidor es mayor a la presentada por el sustrato.

Tanto el valor de la velocidad máxima de reacción (Vmáx) como el de la constante de Michaelis (Km), disminuyen para el caso de la enzima con inhibidor, lo que indica que el inhibidor, en este caso el clorpirifos, se une al complejo ES (enzima-sustrato) e interfiere en la formación del producto, produciendo una disminución en ambas constantes cinéticas. El tipo de inhibición se denomina incompetitiva o acompetitiva, en donde el inhibidor se une a un punto diferente del sitio activo de la enzima (Voet et al., 2007). La adición del inhibidor produce una disminución en el valor de Vmax lo que indica que la enzima tarda más tiempo en realizar su función catalítica.

La constante de inhibición (Ki) fue 5.70E-08 M, que se determinó mediante la ecuación 4.

$$
k m(i)=k m+\frac{k m[I]}{K i}
$$

Donde $[\mathrm{I}]=$ corresponde al valor de la concentración de clorpirifos utilizado en $\mathrm{M}$ (5.70E-08). El valor de ki encontrado es muy bajo, lo que representa que el inhibidor es potente y la unión que este realiza en el complejo ES es fuerte, por ende, dificulta que la reacción sea reversible.

Con respecto a la inmovilización de elemento biológico, los tratamientos 1 y 2 fueron evaluados en los electrodos de carbono, platino, oro, carbono-azul de prusia, carbono-ferrocianuro, y los tratamientos 3 y 4 se evaluaron en los electrodos de carbono, platino y oro.

La voltametría cíclica se realizó con el fin de encontrar el voltaje óptimo de oxidación de la tiocolina sobre cada tipo de electrodo, evaluando en cada uno la inmovilización enzimática con los cuatro tratamientos aplicados. Según el material del electrodo de trabajo, la tiocolina, producto de la hidrólisis de la acetiltiocolina, 
se oxida en la superficie del electrodo a un voltaje característico, lo que conlleva a un aumento de la corriente de salida. En la voltametría cíclica dicho aumento de corriente se observa como un pico que indica la actividad enzimática (Andreescu y Marty, 2006). En la figura 3 se muestra la forma característica del pico correspondiente a la actividad enzimática. De la misma gráfica se puede obtener el valor del voltaje (eje $\mathrm{x}$ ) y la corriente que se alcanza (eje y) en la presencia del pico, determinando de este modo el valor del voltaje en donde se oxida la tiocolonila sobre el electrodo de trabajo.

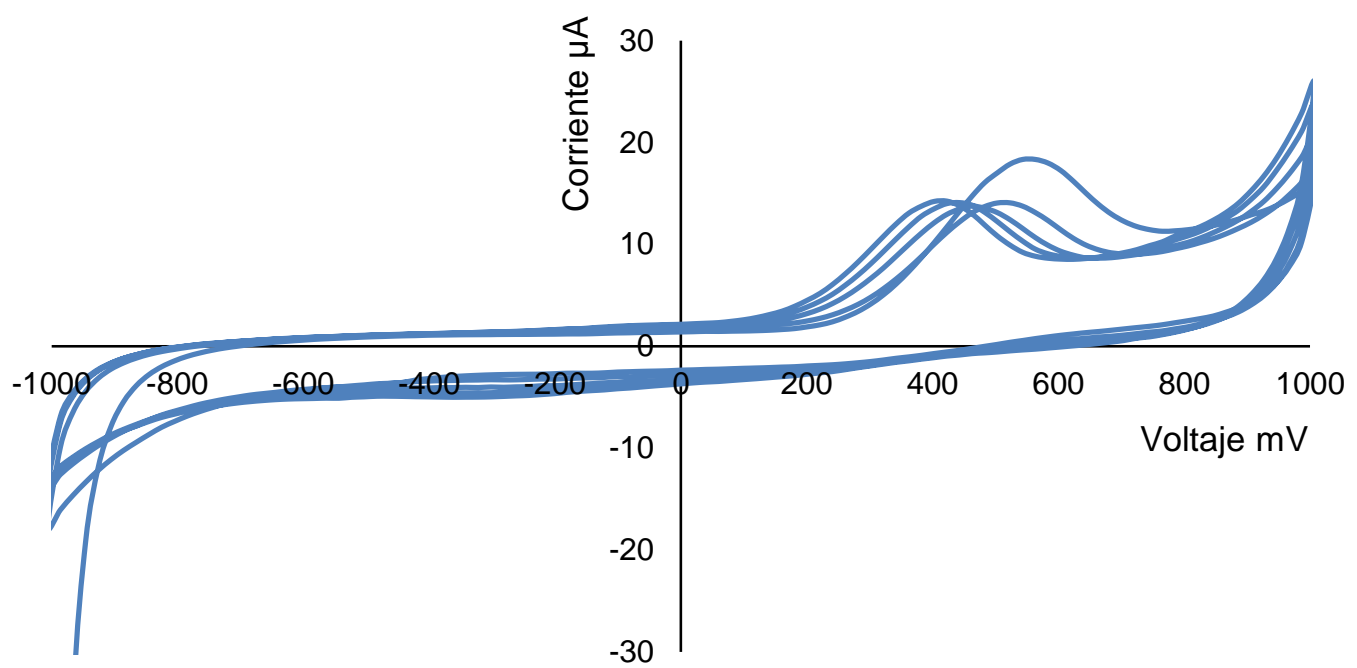

Fig. 3: Gráfica representativa de la Voltametría cíclica con presencia de actividad enzimática.

En los ensayos realizados se obtuvo que el electrodo de oro no presentó respuesta (no hubo presencia de pico) claramente positiva para la inmovilización enzimática con alguno de los cuatro tratamientos aplicados. Múltiples autores afirman que los electrodos de oro pueden presentar interferencias atribuidas a su fabricación cuando son utilizados para el diseño de biosensores enzimáticos (Li et al., 2012; Kadara, et al., 2009), por ende, en la mayoría de los casos para su buen funcionamiento se requiere de la modificación de su superficie mediante pretratamiento del electrodo (Arduini et al., 2013). En cuanto al electrodo de platino, presentó un pico en valores de voltaje muy cambiantes para cada uno de los ensayos en los cuatro tratamientos, dando como resultado valores de la desviación estándar correspondiente a $46 \%, 111 \%, 58 \%$ y $30 \%$ para los tratamientos 1, 2, 3 y 4, respectivamente, por ende, las pruebas realizadas con este electrodo para todos los tratamientos fueron descartadas por no presentar repetibilidad entre los ensayos.

Al comparar los resultados con los ensayos del blanco para los electrodos de carbono, carbono con ferrocianuro y carbono con azul de Prusia, se tomó la decisión de seleccionar al tratamiento 1 como el más óptimo para el electrodo de carbono y el electrodo de carbono-azul de prusia por que mostró menos dispersión de los datos $(0.93 \%)$. Para el electrodo de carbono con ferrocianuro fue seleccionado el tratamiento 2 , presentando la menor desviación de los datos $(0.47 \%)$. El tratamiento 4 por la alta desviación de los datos fue descartado como una opción de trabajo. Los resultados para la inmovilización de la enzima en donde se presentó el mejor pico de actividad enzimática en los diferentes tipos de electrodos se resumen en la tabla 1.

Tabla 1: Resultados positivos de la evaluación de tratamientos de inmovilización en varios tipos de electrodos.

\begin{tabular}{|c|c|c|c|}
\hline Tipo de Electrodo & Tratamiento con mejor respuesta & Voltaje a usar $(\mathrm{mV})$ & $\begin{array}{c}\text { Corriente } \\
\text { promedio }(\mu \mathrm{A})\end{array}$ \\
\hline Carbón & Tratamiento 1 & 460 & 14.46 \\
\hline Carbon-azul de Prusia & Tratamiento 1 & 400 & 16.26 \\
\hline Carbón-Ferrocianuro & Tratamiento 2 & 400 & 11.07 \\
\hline
\end{tabular}

Con el objetivo de mejorar la respuesta del biosensor se realizó voltametría cíclica a diferentes concentraciones de sustrato en los tres tipos de electrodos seleccionados (carbón, carbon-azul de prusia, carbón-ferrocianuro) se evidenció que la mejor respuesta del electrodo se obtuvo para una concentración de sustrato de $5 \mathrm{mM}$. Las características de los electrodos usados para los ensayos de inhibición en agua, leche cruda y leche descremada (Figura 4) fueron: (i) Electrodo de carbono: inmovilización enzimática con 2.5\% GA, 6\% BSA y 100U AChE, concentración de sustrato de $5 \mathrm{mM}$ en los ensayos de inhicición; (ii) Electrodo de carbon-prussian blue: inmovilización enzimática con $2.5 \%$ GA, $6 \%$ BSA y $100 \mathrm{U}$ AChE, concentración de sustrato de $5 \mathrm{mM}$ en los ensayos de inhicición; y (iii) Electrodo de carbono-ferrocianuro: inmovilización enzimática con $2.5 \%$ GA, $6 \%$ BSA y $10 \mathrm{U}$ AChE, concentración de sustrato de $5 \mathrm{mM}$ en los ensayos de inhicición . 

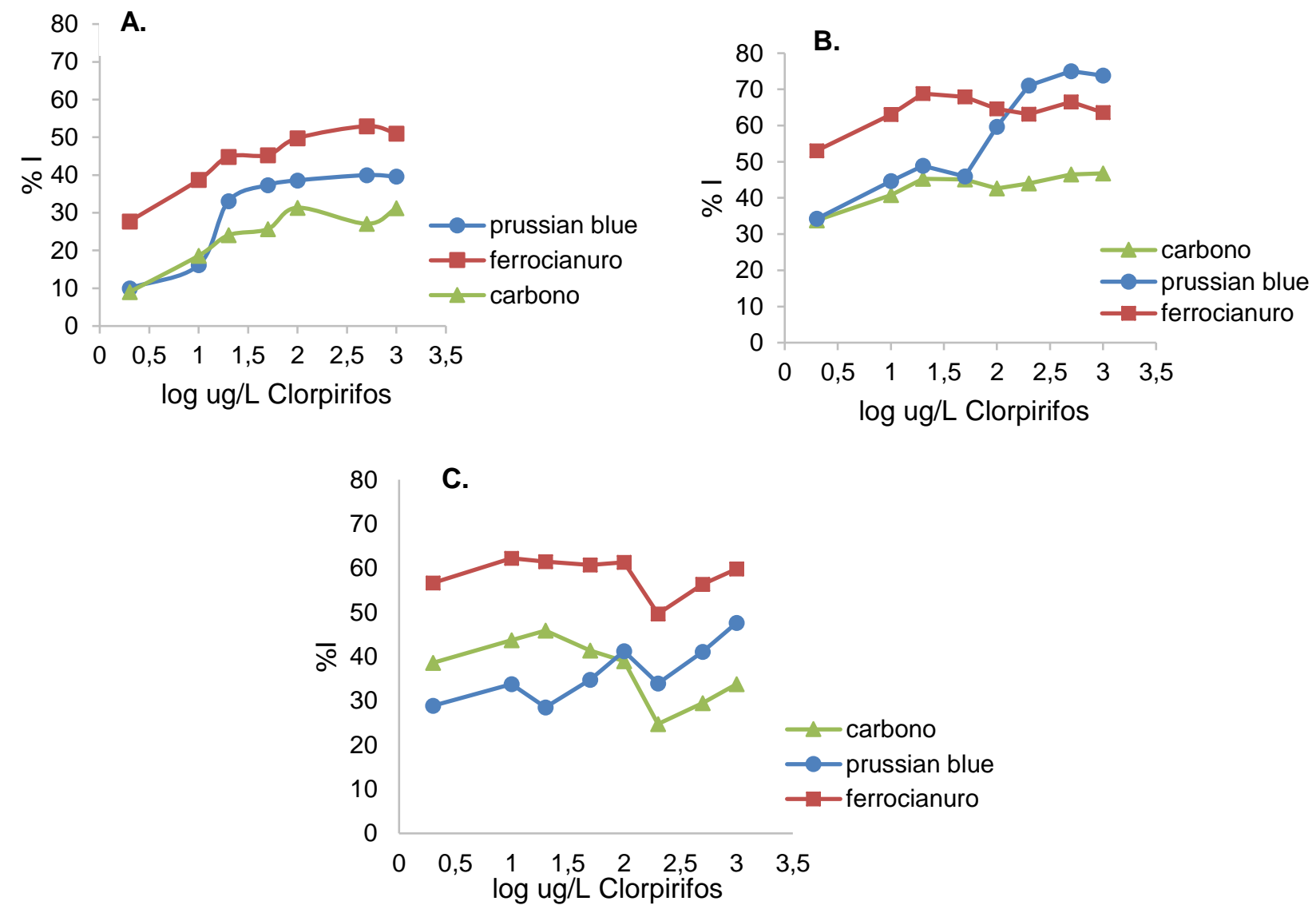

Fig. 4: Ensayos de inhibición del clorpirifos en diferentes matrices. A. Efecto del clorpirifos en solución acuosa, B. Efecto del clorpirifos en la matriz de leche cruda, C. Efecto del clorpirifos en la matriz de leche descremada.

En la inmovilización, la adición a lo último del glutaraldehido mejoró la respuesta de la actividad enzímatica. Silva et al. (2004) en su trabajo mencionan que el glutaraldehido puede comunmente actuar inhibiendo la enzima y para controlar esto debe ser adicionado de último para elaborar la mezcla de la inmovilización, y utilizar concentraciones altas de enzima. Así se explica que la mayoría de los tratamientos óptimos para la inmovilización resultaron para una concentración de enzima de 100U. Según la figura 4 A, se presentó una evidente respuesta a la inhibición del pesticida utilizando una matriz de agua en los tres tipos de electrodos de carbón utilizados, pero la mejor respuesta se obtuvo para el electrodo de carbono con ferrocianuro, el cual muestra porcentajes de inhibición más elevados.

Según los resultados para la leche cruda (figura 4 B), el biosensor resultó ser sensible a la concentración del inhibidor, ya que los porcentajes de inhibición para una concentración tan pequeña de clorpirifos, como $2 \mu \mathrm{g} / \mathrm{L}$, son altos (mayor al 30\%) para los tres tipos de electrodos, lo que demuestra una alta sensibilidad en el instrumento de medida. Para los tres electrodos se demuestra una linealidad en los resultados del porcentaje de inhibición hasta el punto tres de la curva correspondiente a $20 \mu \mathrm{g} / \mathrm{L}$ de clorpirifos, valor correspondiente al límite permitido por la OMS para la leche de vaca. Por tanto, el biosensor, según estos resultados, puede ser útil para detectar valores por debajo de este límite, que era lo que se pretendía en este trabajo de investigación. Este resultado es una mejora considerable a los límites de detección reportados en Rodríguez et al.( 2013) y Morales et al ( 2010) (del orden de $1000 \mu \mathrm{g} / \mathrm{L}$ ) y coincide con el límite de detección, obtenido con un biosensor fluorescente, en Silleti et al. (2015). La mejora es atribuible a la mejor selección de tratamientos de inmovilización y electrodos.

Es evidente que existen diferencias en los resultados obtenidos en agua y en la leche cruda, lo que demuestra un efecto matriz en el método de análisis. Al igual que en la matriz de agua, las señales de inhibición más intensas para la matriz de leche cruda utilizando el biosensor amperométrico se obtuvieron utilizando el electrodo de carbono con ferrocianuro como potencializador electroquímico. De manera similar que, para la leche cruda, para la leche descremada se evidencia un fuerte incremento en los valores obtenidos del porcentaje de inhibición (figura $4 \mathrm{C}$ ) con respecto a los obtenidos para el ensayo del biosensor en agua, evidenciando un posible efecto matriz en el método. Aparentemente la señal del biosensor no se ve afectada por la grasa, ya que la señal de la corriente medida como porcentaje de inhibición denota una alta respuesta 
tanto en leche cruda como leche descremada, para concentraciones muy bajas del inhibidor como $2 \mu \mathrm{g} / \mathrm{L}$. Al igual que para el agua y la leche cruda, la inhibición más intensa para la leche descremada utilizando el biosensor amperométrico, se obtuvo utilizando el electrodo de carbono con ferrocianuro. Para la validación del biosensor como instrumento de detección del clorpirifos se evaluaron los criterios de linealidad, precisión, exactitud, especificidad y efecto matriz en el electrodo de carbón con ferrocianuro en matriz de leche cruda.

Linealidad: La guía SANTE 11945/2015 establece que para que se cumpla linealidad, los residuales deben tener un valor menor o igual al $20 \%$, para este caso no se cumple con dicho criterio, los residuales exceden dicho valor por tanto la técnica no es lineal.

Precisión, Exactitud y Especificidad: El criterio para que la precisión se cumpla, es que el \%RSD para el límite de cuantificación (LC) establecido en $10 \mu \mathrm{g} / \mathrm{L}$ debe ser menor o igual al $20 \%$. El resultado obtenido fue de un $\% R S D=19.99$ lo que demuestra que el método es preciso. Por otro lado, para que el método sea exacto debe cumplir, según la guía SANTE 11945/2015, que el porcentaje de recuperación de la matriz enriquecida con el valor del LC debe estar dentro del rango 70-120\%. Con el biosensor, el porcentaje de recuperación fue $59.88 \%$, por ende, el método no es exacto. La especificidad se evaluó realizando un análisis de blancos, para lo cual el valor debe ser menor al equivalente al $30 \% \mathrm{LC}$, para este caso el valor promedio del blanco fue $25.89 \%$ LC, demostrándose que el método es específico.

Efecto matriz: Para determinar el efecto matriz se comparó la pendiente de la curva del analito en matriz (leche cruda), con la pendiente de la cuerva del analito en agua, obteniéndose un porcentaje de diferencia entre ambas pendientes de $35.71 \%$, por lo tanto, el método posee un efecto matriz ya que la guía SANTE $11945 / 2015$ establece que hay efecto matriz si dicha diferencia es mayor al $15 \%$

Para la validación de la técnica de cromatografía de gases, de manera similar que en el biosensor, fueron evaluados los siguientes parámetros:

Linealidad: Se tomó como punto de partida un intervalo de concentraciones (Tabla 2) de acuerdo con la finalidad de este trabajo, de tal forma que el valor máximo de concentración de clorpirifos en leche cruda de vaca corresponde a $20 \mu \mathrm{g} / \mathrm{L}$, valor establecido por la Organización Mundial de la Salud. El valor de los residuales fue menor o igual al 20\%, para todos los puntos de la curva de calibración (Tabla 2), por tanto, la técnica es Lineal.

Tabla 2: Resultados promedio para los ensayos de Linealidad.

\begin{tabular}{|c|c|c|c|}
\hline $\begin{array}{c}\text { Valor de matriz teórico } \\
(\mu \mathrm{g} / \mathrm{L})\end{array}$ & $\begin{array}{c}\text { Valor experimental } \\
\text { promedio }(\mu \mathrm{g} / \mathrm{L})\end{array}$ & $\mathrm{s}$ & $\begin{array}{c}\text { Residuales promedio } \\
(\%)\end{array}$ \\
\hline 5 & 4.54 & 0.31 & 9.26 \\
\hline 10 & 10.87 & 0.34 & 8.71 \\
\hline 20 & 19.78 & 1.22 & 4.24 \\
\hline 30 & 30.79 & 2.45 & 5.39 \\
\hline 50 & 50.39 & 1.45 & 2.08 \\
\hline 70 & 69.12 & 6.49 & 6.51 \\
\hline 100 & 99.52 & 6.29 & 4.98 \\
\hline
\end{tabular}

Precisión, Exactitud y Especificidad: Se realizaron nueve repeticiones $(n=9)$ en el valor establecido como el límite de cuantificación $(10 \mu \mathrm{g} / \mathrm{L})$ y dos veces el límite de cuantificación $(20 \mu \mathrm{g} / \mathrm{L})$. El criterio para que la precisión se cumpla, que el \%RSD sea menor o igual al $20 \%$. La exactitud se evaluó también con un $n=9$ pata el límite de cuantificación y dos veces el límite de cuantificación; estableciéndose en función del porcentaje de recuperación, el cual debe estar en el rango $70-120 \%$.

Para la especificidad se realizó un análisis en blanco de matriz en el que la cantidad de analito no debe superar el 30\% del Límite de cuantificación. En la tabla 3 se muestra los resultados de la evaluación de precisión, exactitud y especificidad. En la tabla, LC representa el Límite de cuantificación y 2LC representa dos veces el límite de cuantificación. El método es preciso ya que el \%RSD es menor al 20\% para cada ensayo, es exacto por que el porcentaje de recuperación de 2 LC estuvo en el rango $70-120 \%$ y específico porque la cuantificación del blanco de matriz fue 3.70\% comparado con el límite de cuantificación. 
Tabla 3: Resumen de los datos obtenidos para los ensayos de precisión, exactitud y especificidad

\begin{tabular}{|c|c|c|c|}
\hline Concentración teórica & $10 \mu \mathrm{g} / \mathrm{L}(\mathrm{LC})$ & $20 \mu \mathrm{g} / \mathrm{L}(2 \mathrm{LC})$ & Blanco matriz \\
\hline $\bar{x}$ & $7.76 \mu \mathrm{g} / \mathrm{L}$ & $16.54 \mu \mathrm{g} / \mathrm{L}$ & $0.37 \mu \mathrm{g} / \mathrm{L}$ \\
\hline$S$ & 1.39 & 2.90 & 0.65 \\
\hline$\%$ RSD & 17.90 & 17.51 & No aplica \\
\hline \% Recuperación & 77.63 & 82.68 & No aplica \\
\hline
\end{tabular}

Efecto matriz: Se realizó una comparación entre la curva de calibración en solvente (acetonitrilo) y la curva de calibración en matriz (leche). Se obtuvo una pendiente de 0.72 y 619.60 , respectivamente, cuyo $\% E M=85955.55$ Luego existe un notorio efecto matriz en el método, por tanto, la curva de calibración debe realizarse en la matriz cuando se vaya a realizar la lectura de muestras mediante cromatografía de gases.

\section{CONCLUSIONES}

De los resultados obtenidos, su análisis y discusión, se pueden obtener las siguientes conclusiones sobre el uso del biosensor enzimático para la detección de plaguicidas en leche:

1) El pesticida clorpirifos actúa como un inhibidor de tipo acompetitivo, produciendo una disminución en el valor de la velocidad máxima y la constante de Michaelis-Menten de la enzima acetilcolinesterasa.

2) Los resultados óptimos para la inmovilización enzimática mediante "cross linking" se obtuvieron utilizando cantidades grandes de concentración de enzima sobre electrodos de carbono.

3) El biosensor enzimático amperométrico presentó un rango de detección del analito comprendido entre 5 $\mu \mathrm{g} / \mathrm{L}$ y $1000 \mu \mathrm{g} / \mathrm{L}$

4) En el agua, leche cruda y leche descremada, el electrodo carbón con ferrocianuro fue el que presentó un mayor porcentaje de inhibición y por lo tanto una mayor sensibilidad en la detección del plaguicida.

5) No se evidenció una interferencia de la señal de la corriente eléctrica por la composición de la grasa en la leche.

6) La técnica de biosensores para la determinación de clorpirifos en leche puede servir como una técnica cualitativa de detección del pesticida, pero no cuantitativa. Los biosensores pueden ser herramientas de análisis capaces de detectar la presencia de clorpirifos en leches en tiempo real y generar alarmas. Para la cuantificación del clorpirifos se requiere una técnica más robusta como la cromatografía de gases.

7) El biosensor desarrollado y el prototipo electrónico conforman un sistema completo de medida para ser empleado directamente en hatos lecheros y permitir obtener alarmas tempranas de contaminación. Posteriormente, las muestras positivas pueden ser sometidas a cromatografía para obtener mediciones precisas y certificadas.

\section{AGRADECIMIENTOS}

A la vicerrectoría de investigaciones de la U. de Antioquia por la financiación del proyecto CODI PRG-2014-462.

\section{REFERENCIAS}

Andreescu, S. y J.L. Marty, Twenty Years Research in Cholinesterase Biosensors: From Basic Research to Practical Applications, Biomolecular Engineering, 23(1), 1-15 (2006)

Arduini, F., S. Guidone y otros tres autores, Acetylcholinesterase Biosensor Based on Self-assembled Monolayer-modified Gold-screen Printed Electrodes for Organophosphorus Insecticide Detection, Sensors y Actuators: B, Chemical, 179, 201208 (2013)

AOAC Official Method 2007.01, Pesticide Residues in Foods by Acetonitrile Extraction and Partitioning with Magnesium Sulfate, Gas Chromatography/Mass Spectrometry and Liquid Chromatography / Tandem Mass Spectrometry, First Action (2007)

Asociación de procesadores de la leche, Asoleche, Aumento en el Consumo de Lácteos en Colombia en 2016 (2013)

Buck, W. B., G. D. Osweiler y G. A. Van Gelder, Toxicología Veterinaria Clínica y Diagnóstica, Editorial Acribia. Zaragoza, España, pp. 475 (1981)

Cano, J.B., K. Buonasera y G. Pezzotti, Transduction Methods Used on Biosensors: Amperometry and Fluorescence. Revista Facultad de Ingeniería Universidad de Antioquia, (72), 104-115 (2014) 
Cano J.B., D. Giannini, G. Pezzotti, G. Rea y M.T. Giardi, Space Impact and Technological Transfer of a Biosensor Facility to Earth Application for Environmental Monitoring, Recent Patents on Space Technology, 1(1), 18-25(8) (2011)

Ellman, G.L., K.D. Courtney, V. Andres y R.M. Featherstone, A New and Rapid Colorimetric Determination of Acetylcholinesterase Activity, Biochemical Pharmacology, 7, 88-95 (1960)

Engel, L.S., D.A. Hill y otro ocho autores, Pesticide Use and Breast Cancer Risk Among Farmers' Wives in the Agricultural Health Study, Am. J. Epidemiol., 161(2), 121-35 (2005)

Ferri, D., P. Gaviña y otros cuatro autores, Detection and Discrimination of Organophosphorus Pesticides in Water by Using a Colorimetric Probe Array, Sensors and Actuators B, Chemical, 202, 727-731 (2014)

Food Standars FAO / WHO Pesticide Residues in Food and Feed, Maximum Residue Limits for Chlorpyrifos (2013)

Hernández, M., C.A. Galán, G.A. Álvarez y E. Páez, Desarrollo de un Biosensor Amperométrico en Configuración plana para la Cuantificación de Colesterol, doi: 10.4067/S0718-07642011000600004, Información Tecnológica, 22(6), 25-32 (2011)

Kadara, R.O., N. Jenkinson y C.E. Banks, Characterization and Fabrication of Disposables Screen Printed Microelectrodes, Electrochem. Commun., 11,13-77 (2009)

Lavecchia, T., A. Tibuzzi y M. T. Giardi, Biosensors for functional food safety and analysis. In Bio-farms for nutraceuticals, pp. 267-281, Springer, Boston, MA. (2010)

Li, M., Y.T. Li, D.W. Li y Y.T. Long, Recent Developments and Applications of Screen-printed Electrodes in Environmental Assays-A review. Anal. Chim. Acta, 734, 19-31 (2012)

Montañez, J.L., E.G. Ramos, S. Alegret y R.J. Delgado, Biosensor de Glucosa basado en un Biocompósito disperso de GrafitoEpoxi-Platino-Glucosa Oxidasa, doi: 10.4067/S0718-07642011000100005, Información Tecnológica, 22(1), 29-40 (2011)

Morales, C., N. Rodríguez, L. F. Restrepo y C. López, Relación entre Residuos de Clorpirifos en Leche y Sangre de Vacas Holstein y Niveles Séricos de Estradiol y Tiroxina, Revista electrónica de Veterinaria, 11(1), 1-22 (2010)

Rodríguez, D.C., S. Carvajal y G. Peñuela, Effect of Chlorpyrifos on The Inhibition of the Enzyme Acetylcholinesterase by Cross-linking in Water-supply Samples and Milk from Dairy Cattle, Talanta, 111, 1-7 (2013)

SANTE / 11945, Guidance Document on Analytical Quality Control and Method Validation Procedures for Pesticides Residues Analysis in Food and Feed (2015)

Scognamiglio, V., D. Raffi y otros cinco autores, Chlamydomonas Reinhardtii Genetic Variants as Probes for Fluorescence Sensing System in Detection of Pollutants, Analytical and Bioanalytical Chemistry, 394(4) (2009)

Scognamiglio, V., I. Pezzotti y otros cinco autores, Towards an Integrated Biosensor Array for Simultaneous and Rapid Multi-analysis of Endocrine Disrupting Chemicals, Analytica chimica acta, 751, 161-170 (2012)

Silleti S., G. Rodio y otros cinco autores, An Optical Biosensor Based on a Multiarray of Enzymes for Monitoring a Large Set of Chemical Classes in Milk, Sensors and Actuators B: Chemical, doi: 10.1016/j.snb.2015.03.092, 215, 607-617 (2015)

Silva Nunes, G., G. Jeanty y J.L. Marty, Enzyme Immobilization Procedures on Screen-printed Electrodes Used for the Detection of Anticholinesterase Pesticides, Analytica Chimica Acta, 523(1), 107-115 (2004)

Tecles, F., C. Gutiérrez-Panizo, S. Martínez Subiela y M.D. Parra, Comparación de la 2,2'-ditiodipiridina y el ácido 5,5,'-ditiobis- 41 2-nitrobenzoico en la Determinación de Colinesterasa en Sangre Entera de Perro. An. Vet. (Murcia) $16,41-54,(2000)$

Voet, D., J. Voet y C. Pratt, Fundamentos de Bioquímica. La Vida a Nivel Molecular, Madrid, España, Editorial Médica Panamerican S.A. (2007)

Whitford, F., D. Pike y otros cuatro autores, The Benefits of Pesticides. A Story Worth Telling, Purdue Extension PPP-70, 1-33 (2009) 